\title{
REGEPE

\section{PROPOSIÇÃO DE UM MODELO DE GERENCIAMENTO DE PROJETOS PARA PEQUENAS E MÉDIAS EMPRESAS (PME)}

\section{Resumo}

1 Rodrigo Menezes de Souza 2 Taina Alves dos Santos

3 Emerson Antonio Maccari 4 Marcos Rogerio Mazieri

Objetivo do estudo: propor um modelo de gerenciamento de projetos adequado ao contexto de pequenas e médias empresas (PME).

Metodologia/abordagem: por meio de estudo bibliométrico, associado à análise lexical, com classificação hierárquica descendente (CHD), foram realizados levantamentos de publicações sobre o tema e de inferências estatísticas dos textos, a fim de encontrar padrões de correspondência entre as palavras e as frases, para analisar, com clareza, os conceitos envolvidos.

Principais resultados: a pesquisa permite entender melhor o gerenciamento de projetos nas PME, pois destacou, dos modelos utilizados, características relativas à inovação, ao foco no cliente, às pessoas, à desburocratização, à flexibilidade e à gestão por profissionais não especialistas. A conclusão apontou a necessidade de simplificação e de baixo custo para a implementação desses modelos em tais organizações.

Contribuições teóricas/metodológicas: este estudo contribuiu com a evolução da literatura e do modelo pré-existente, de Kozlowski e Matejun (2016), oferecendo aos sócios proprietários de PME a clareza na implementação de novos projetos.

Relevância/originalidade: são relevantes a descoberta de novas características, de processos e de benefícios, o que possibilita às PME se antecipar aos problemas; e o desenvolvimento de competências no gerenciamento de projetos e na gestão do negócio.

Contribuições sociais/para a gestão: a aplicação de um modelo de gerenciamento de projetos, para PME, flexível a diversos contextos e motivações, agregando valor a essas organizações, em termos de planejamento, de execução e de clareza dos resultados.

Palavras-chave: Gerenciamento de projetos. Pequenas e médias empresas. Bibliometria. Análise lexical. Classificação Hierárquica Descendente.

Recebido: 19/11/2019 / Aprovado: 12/02/2020

Editor responsável: Profa. Dra. Vânia Maria Nassif

Processo de avaliação: Double Blind Review

Doi: https://doi.org/10.14211/regepe.v9i3.1475

\footnotetext{
${ }^{1}$ Universidade Nove de Julho - UNINOVE, São Paulo, (Brasil). E-mail: rodrigo@drinova.com Orcid id: https://orcid.org/0000-0002-6886-0539

2Universidade Nove de Julho - UNINOVE, São Paulo, (Brasil). E-mail: taina.alves@uni9.edu.br Orcid id: https://orcid.org/0000-0002-5182-4312

3Universidade Nove de Julho - UNINOVE, São Paulo, (Brasil). E-mail: emerson.maccari@gmail.com Orcid id: https://orcid.org/0000-0001-7085-224X

${ }^{4}$ Universidade Nove de Julho - UNINOVE, São Paulo, (Brasil). marcosmazzzieri@gmail.com Orcid id: https://orcid.org/0000-0003-1338-3912
} 


\section{PROPOSING A PROJECT MANAGEMENT MODEL FOR SMALL AND MEDIUM-SIZED ENTERPRISES (SMES)}

\section{Abstract}

Objective: to propose a project management model suitable for the context of small and medium-sized enterprises (SME).

Methodology/approach: through a bibliometric study associated with a lexical analysis with Descending Hierarchical Classification (DHC), this analysis allowed for statistical inferences to be made regarding the texts, to find patterns between words and phrases that clarified the concepts involved.

Main results: the research contributes to the understanding of project management in SME context, expanding the focuses on innovation, customers, employees, flexibility, and management performed by non-specialists. The conclusion pointed out the need for simplification and low cost for the implementation of these models in such organizations.

Theoretical/Methodological Contributions: this study contributed to the evolution of the literature and the pre-existing model by Kozlowski and Matejun (2016), offering the owners of SMEs the clarity in the implementation of new projects.

Relevance/Originality: the discovery of new characteristics, processes and benefits is relevant, enabling SMEs to anticipate problems; and the development of skills in project management and business management.

Social/management contributions: the application of a project management model, for SMEs, flexible to various contexts and motivations, adding value to these organizations, in terms of planning, execution and clarity of results.

Keywords: Project management. Small and medium-sized enterprises. Bibliometric. Lexical Analysis. Descending Hierarchical Classification. 


\section{PROPUESTA DE MODELO DE GESTIÓN DE PROYECTO PARA PEQUEÑA Y MEDIANA EMPRESA (PYME)}

\section{Resumén}

Objetivo del estudio: esta investigación tuvo como objetivo proponer un modelo de gestión de proyectos adecuado al contexto de las pequeñas y medianas empresas (PYME).

Metodología/enfoque: se realizó una encuesta general de las publicaciones sobre el tema por medio de un estudio bibliométrico asociado con un análisis léxico con Clasificación jerárquica descendente. El análisis léxico también permitió realizar inferencias estadísticas sobre los textos, a fin de encontrar patrones de correspondencia entre palabras y frases para que trajeron claridad cuanto a los conceptos involucrados.

Resultados principales: la investigación contribuyó para la comprensión de la gestión de proyectos en las PYME, presentando características de estos modelos centrados en la innovación, en el enfoque al cliente, a las personas, flexibilidad y la gestión por parte de personas que no son especialistas. Además de la premisa de que los modelos de gestión de proyectos para tales organizaciones deben ser simplificados y con bajo costo de implementación.

Contribuciones teóricas/metodológicas: los resultados permitieron la evolución de la literatura y el modelo preexistente de Kozlowski y Matejun (2016), permitiendo que los proyectos se implementen en las PYME claramente para los empresarios.

Relevancia/Originalidad: nuevas características, procesos y beneficios fueron descubiertos, permitiendo a las PYME anticipar problemas y desarrollar habilidades de gestión de proyectos y de su negócio.

Contribuciones sociales/de gestión: la gestión de proyectos es algo presente en la realidad de las PYME. Por esta razón los resultados de este estudio ayudan a las PYME a aplicar un modelo flexible a los diversos contextos y motivaciones inherentes a este tipo de organizaciones. Este modelo ofrece valor en términos de planificación, ejecución y claridad de resultados.

Palabras clave: Gestión de proyectos. Pequeñas y medianas empresas. Bibliometría. Análisis léxico. Clasificación jerárquica descendente. 


\section{INTRODUÇÃO}

Na história, pequenas e médias empresas (PME) antecederam o surgimento das grandes corporações, movimentando a economia dos países. Por essa razão, estudos sobre elas foram iniciados justamente nessa área do conhecimento (Leone, 1991). O impacto na geração de empregos e o reconhecimento do papel das PME, no surgimento de novos produtos para a economia global, desde a década de 1990, têm atraído a atenção e a competitividade para essas organizações (Subrahmanya, 2004; Turner, Ledwith, \& Kelly, 2010). No Brasil, elas respondem por 44\% dos empregos formais criados e, ao menos, 27\% do produto interno bruto (PIB) (Sebrae, 2014).

PME são organizações mais sensíveis aos abalos econômicos, pois atuam em mercados de tamanho limitado, com forte concorrência, e em constante instabilidade financeira (Almeida, Costa, Braga Junior, \& Porto, 2018; Köksal \& Orman, 2014; Serrasqueiro \& Caetano, 2015). Esses fatores podem ocasionar restrições, sob diversas perspectivas, como em relação à capacidade de investimento (Cohen \& Levinthal, 2009; Dobbs \& Hamilton, 2007). Isso explica as inúmeras preocupações acerca do retorno dos investimentos realizados e do fluxo de caixa (Adair \& Adaskou, 2015; Kozlowski \& Matejun, 2016; Subrahmanya, 2004) nessas organizações.

Os processos de transformação digital e de globalização impactaram a economia das PME, sobretudo em países em desenvolvimento, impondo-Ihes a necessidade de modernizar seus processos e de utilizar novas tecnologias digitais, alterando, assim, sua perspectiva de criação de valor na busca pela competitividade (Subrahmanya, 2004; Vial, 2019).

Em uma economia industrializada, as PME têm papel fundamental, pois preenchem lacunas deixadas pelas grandes. Então, qualquer iniciativa nova, que exige decisão de investimento, deve ter, de forma clara e racional, o entendimento de todas as variáveis capazes de afetar o crescimento da empresa. Entre elas, estão os benefícios econômicos (Jenkins, 2006, 2009), a qualidade de gerenciamento, os consumidores, as políticas, as influências internas e externas, os processos de aprendizagem, as tomadas de decisões (Dobbs \& Hamilton, 2007) e a transformação digital (Vial, 2019).

Os empreendedores em PME precisam desenvolver competências estratégicas para avaliar, planejar e executar ações que mantenham a empresa em crescimento (Tehseen, Ahmed, Qureshi, Uddin, \& Ramayah, 2019). Dessa forma, o gerenciamento 
de projetos, voltado a essas organizações, propõe-se a esclarecer, de forma racionalizada, o entendimento dos líderes a respeito dos benefícios a serem alcançados por novas iniciativas (Kozlowski \& Matejun, 2016). Contudo, são ainda incipientes os estudos que exploram as habilidades das PME em gerenciar projetos, tampouco os que conectam suas atividades funcionais às diversas áreas de gerenciamento (Kozlowski \& Matejun, 2016) ou que o relacionam à lucratividade (Pollack \& Adler, 2016).

Há duas perspectivas claras quanto ao uso do gerenciamento de projetos para os líderes das PME: (1) a sua associação ao aumento de receita; e (2) a diminuição do percentual de líderes que o associam ao decréscimo da lucratividade (Pollack \& Adler, 2016). Tal fato corrobora os papéis determinantes da gestão de projetos (GP), que são: atender às motivações e promover o engajamento dos proprietários dessas organizações.

Uma das necessidades mais recorrentes das PME é a obtenção de recursos financeiros para investimento (Serrasqueiro \& Caetano, 2015); e, mesmo que eles consigam ser captados, nem todas as empresas sabem utilizá-los de forma adequada, principalmente se elas não tiverem técnicas de gerenciamento compatíveis com seu porte. Como resultado, elas acabam incorrendo no risco de necessitar de mais investimentos, gerando, com isso, um ciclo de restrições financeiras não resolvidas.

Esta pesquisa, então, propõe um modelo de gerenciamento de projetos adequado ao contexto das PME, com o objetivo principal de auxiliar as empresas na compreensão das características, dos processos e dos benefícios a serem alcançados.

\section{REFERENCIAL TEÓRICO}

O referencial teórico deste estudo fundamentou-se na contextualização das características gerais e de gestão das PME, além do gerenciamento de projetos, aplicado nessas organizações.

\subsection{CARACTERÍSTICAS GERAIS DAS PME}

A partir de uma revisão da literatura sobre os estudos que explicam o crescimento das PME, para auxiliar no entendimento dos diversos contextos nos quais essas organizações estão inseridas e se desenvolvem, Dobbs e Hamilton (2007) propõem uma visão pautada nas seguintes perspectivas: estocástica, descritiva, 
evolucionária, visão baseada em recursos (RBV), aprendizagem e determinista, cujas características estão discriminadas a seguir (e sintetizadas na Figura 1).

A estocástica explica um conjunto de variáveis, conforme a diversidade das PME (Kozlowski \& Matejun, 2016), responsável por esse crescimento, mas que não tem qualquer relação com o tamanho, no começo da trajetória, e ao longo da história dessas empresas (Dobbs \& Hamilton, 2007).

Na perspectiva descritiva, na qual se enquadra esta pesquisa, verifica-se como as PME rearranjam seus processos internos, para se adaptar às crises, manter-se em crescimento (Dobbs \& Hamilton, 2007) e ter competitividade (Subrahmanya, 2004). O sucesso se dará pela gestão dos recursos financeiros, do pessoal e dos sistemas, a partir das metas do proprietário, sua habilidade operacional, sua capacidade de gerenciamento das demandas existentes, e das estratégias futuras (Lewis, Virginia, \& Churchill, 1983).

Tratando das influências internas e externas, a perspectiva evolucionista ressalta que as PME atuam em pequenos mercados, ou com produtos de nicho específico, não sendo concebível, em termos geográficos e econômicos, a sua atuação em mercados amplos (Leone, 1991).

$\mathrm{Na}$ visão baseada em recursos (RBV), é indicado que, para ter lucratividade, vantagem competitiva e contínuo crescimento, as PME devem repensar suas estratégias de negócio e seus recursos, fazendo as adaptações necessárias.

A aprendizagem, por sua vez, permite aos proprietários de PME, que estão próximos da operação, tomar decisões representativas dos seus valores pessoais e crenças, alinhadas diretamente ao crescimento da organização (Dobbs \& Hamilton, 2007; Bos-Brouwers, 2010; Jamali, Zanhour, \& Keshishian, 2009).

Por fim, a perspectiva determinista envolve estudos estatísticos que, de forma generalista, evidenciam as causas para o crescimento das PME.

Ao longo do seu ciclo de crescimento, as PME percorrem um processo, que se inicia em angariar clientes, e termina na demonstração de sua capacidade de permanência ativa no mercado. Para tanto, a viabilidade de recursos financeiros tem papel crucial até essas empresas alcançarem suas metas e os proprietários decidirem pela expansão ou pela manutenção do tamanho do negócio (Lewis et al., 1983).

Durante esse caminho, nota-se que a implantação de novas estratégias sofre constante influência das aspirações pessoais e das experiências dos proprietários (Dobbs \& Hamilton, 2007). Dessa forma, quaisquer novas iniciativas das PME devem 
ser realizadas, de forma racionalizada, por seus proprietários, para que eles entendam claramente o que será desenvolvido (Jenkins, 2006, 2009; Simpson, Taylor, \& Barker, 2004).

\begin{tabular}{|c|c|c|c|}
\hline Perspectiva & Premissas & $\begin{array}{l}\text { Objeto } \\
\text { do estudo }\end{array}$ & $\begin{array}{c}\text { Variáveis } \\
\text { de crescimento }\end{array}$ \\
\hline Estocástica & $\begin{array}{l}\text { O crescimento da empresa } \\
\text { depende de um conjunto de } \\
\text { variáveis, não relacionadas ao } \\
\text { tempo. }\end{array}$ & $\begin{array}{l}\text { Causas do } \\
\text { crescimento. }\end{array}$ & $\begin{array}{l}\text { Qualidade do } \\
\text { gerenciamento; consumidores; } \\
\text { políticas organizacionais; } \\
\text { outras forças. }\end{array}$ \\
\hline Descritiva & $\begin{array}{l}\text { Como as pequenas empresas } \\
\text { se organizam internamente } \\
\text { para manter o crescimento. }\end{array}$ & $\begin{array}{l}\text { Adaptação para } \\
\text { manter o } \\
\text { crescimento. }\end{array}$ & 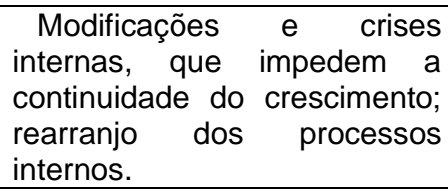 \\
\hline Evolucionária & $\begin{array}{l}\text { O crescimento da organização } \\
\text { é resultado de influências } \\
\text { internas e externas. }\end{array}$ & $\begin{array}{l}\text { Causas do } \\
\text { crescimento. }\end{array}$ & $\begin{array}{l}\text { Influências } \\
\text { externas. }\end{array}$ \\
\hline RBV & $\begin{array}{l}\text { O crescimento da pequena } \\
\text { empresa depende da forma } \\
\text { como seus recursos são } \\
\text { gerenciados para manter a } \\
\text { operação, ao longo do tempo. }\end{array}$ & $\begin{array}{l}\text { Adaptação para } \\
\text { manter o } \\
\text { crescimento. }\end{array}$ & $\begin{array}{l}\text { Estratégias de negócio; } \\
\text { recursos; lucratividade. }\end{array}$ \\
\hline Aprendizagem & $\begin{array}{l}\text { O crescimento da organização } \\
\text { é influenciado pela capacidade } \\
\text { de aprendizado e pela tomada } \\
\text { de decisão de seus gestores. }\end{array}$ & $\begin{array}{l}\text { Adaptação para } \\
\text { manter o } \\
\text { crescimento. }\end{array}$ & 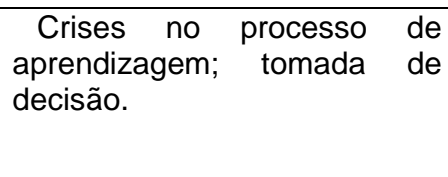 \\
\hline Determinista & $\begin{array}{l}\text { Visão quantitativa dos fatores } \\
\text { relacionados ao ambiente, que } \\
\text { explicam o crescimento da } \\
\text { pequena empresa }\end{array}$ & $\begin{array}{l}\text { Causas do } \\
\text { crescimento. }\end{array}$ & $\begin{array}{cc}\text { Variáveis internas } & \text { e } \\
\text { externas; validação estatística. }\end{array}$ \\
\hline
\end{tabular}

Figura 1. Perspectivas de estudo sobre crescimento das pequenas empresas Fonte: Adaptada de Dobbs e Hamilton (2007).

\subsubsection{CARACTERÍSTICAS DE GESTÃO DE PME}

Nas PME, as inovações são mais constantes, pois elas não têm escala para competir dentro de uma estratégia de preços no mercado (Dobbs \& Hamilton, 2007). Sendo assim, o desenvolvimento de produtos precisa utilizar soluções baratas e de fácil compreensão pelos envolvidos (Schumacher, 1983), pois eles são testados em contato direto com a operação, demandando a necessidade de técnicas de gerenciamento para cuidar adequadamente dos recursos envolvidos (Kozlowski \& Matejun, 2016).

Perspectivas a respeito das características de gestão dos proprietários dessas empresas são importantes, tendo em vista a sua relação próxima da operação e dos funcionários, o que influencia os processos, as estratégias, as tomadas de decisão e 
a geração de resultados (Dobbs \& Hamilton, 2007; Jamali et al., 2009). Por outro lado, as motivações e a liderança, pautadas em decisões de cunho pessoal, por vezes, fazem-nos não investir no crescimento da empresa, quando percebem que o tamanho e os valores esperados (Dobbs \& Hamilton, 2007; Spence, 1999). Por outro lado, essa mesma motivação faz com que os líderes estejam sempre próximos dos funcionários, influenciando os processos e estratégias (Dobbs \& Hamilton, 2007). Isso facilita a tomada de decisão e a geração de resultados, que definem muitas das características comportamentais das organizações (Jamali et al., 2009).

O nível de educação dos empreendedores deve ser considerado, uma vez que, quanto maior a escolaridade, maior a relação que terão com o resultado e a busca pela eficiência do negócio (Dobbs \& Hamilton, 2007). Aliado a isso, a experiência na gestão do negócio representa ganhos, em termos de desenvolvimento operacional, pois permite avanços no modelo de negócios da empresa (Dobbs \& Hamilton, 2007).

Recursos financeiros são fatores cruciais para as PME (Subrahmanya, 2004), gerando constantes preocupações sobre despesas e lucro (Kozlowski \& Matejun, 2016), o que limita, de certo modo, a capacidade de investimento em novas soluções (Dobbs \& Hamilton, 2007). Muitas vezes, isso demanda que essas organizações realizem processos de cooperação e novas alianças (Almeida et al., 2018; Breu, Guggenbichler, \& Wollmann, 2008; Cohen \& Levinthal, 2009), bem como a flexibilização de suas estratégias (Dobbs \& Hamilton, 2007), em busca de responder às pressões do mercado.

\subsection{GERENCIAMENTO DE PROJETOS EM PEQUENAS EMPRESAS}

Estudos acadêmicos sobre o gerenciamento de projetos têm atraído especial atenção, sobretudo quando a intenção é alinhá-lo aos direcionamentos estratégicos empresariais, que conduzem, por sua vez, ao desenvolvimento de soluções inovadoras (Kwak \& Anbari, 2009), à sua adequação às complexidades inerentes aos novos ambientes de negócios e às relações corporativas (Svejvig \& Andersen, 2015). Nesse sentido, o modelo de gerenciamento de projetos mais recente, encontrado na literatura, é o publicado por Kozlowski e Matejun (2016), que referenciou este estudo (Figura 2).

A implantação de projetos pelas PME possui peculiaridades, que pedem a adequação do gerenciamento ao contexto (Kozlowski \& Matejun, 2016), exigindo versões simplificadas, capazes de imprimir maior agilidade, ter menor custo e manter 
o foco mais nas pessoas do que nos processos burocráticos (Souza, Souza, \& Souza Júnior, 2014; Turner et al., 2012). Nas PME, os motivadores e os objetivos do gerenciamento de projetos se relacionam à busca do aumento de receita ou de mercado, com fins de atender às necessidades de seus proprietários (Kozlowski \& Matejun, 2016). Os projetos, então, são direcionados a uma visão de expectativas, de ações e de aprendizado, além de abarcar os conceitos tradicionais de planejamento, de controle e de validação (Packendorff, 1995).

Nesse cenário, o empreendedor, que exerce o papel de gerente de projeto, tem atuação fundamental na implementação de técnicas simples para a coleta de dados, e nas tomadas de decisão (Jucá Júnior, Conforto, \& Amaral, 2010), sendo que os resultados refletirão o grau dessa participação nos processos (Martens, Belfort, Carneiro, \& Martens, 2014). Nas PME, os recursos humanos são restritos e, por isso, a execução de projetos está sujeita à cooperação limitada, à não utilização de softwares avançados; à comunicação informal (Kozlowski \& Matejun, 2016); e ao gerenciamento por profissionais não especialistas, que, em contrapartida, têm habilidades favorecedoras para a comunicação (Martens et al., 2014), garantindo o apoio dos fundadores do negócio (Turner et al., 2010).

\begin{tabular}{|l|l|l|}
\hline \multicolumn{2}{|c|}{$\begin{array}{c}\text { Fases do } \\
\text { Projeto }\end{array}$} & \multicolumn{2}{|c|}{ Gerenciamento de Projetos em Pequenas Empresas } \\
\hline Planejamento & 1. Motivações e Objeticas de Gerenciamento & Aumento de mercado e de receita. \\
\cline { 2 - 3 } & 2. Gestor do Projeto & $\begin{array}{l}\text { Proprietário da empresa, que possui controle total } \\
\text { sobre as fases de implementação. }\end{array}$ \\
\hline $\begin{array}{l}\text { Execução e } \\
\text { Controle }\end{array}$ & $\begin{array}{l}\text { 3. Gestão de Recursos } \\
\text { Humanos }\end{array}$ & $\begin{array}{l}\text { Depende plenamente dos recursos financeiros } \\
\text { alocados ao projeto; cooperação limitada, prezando } \\
\text { pela ação independente. }\end{array}$ \\
\cline { 2 - 3 } & $\begin{array}{l}\text { 4. Ferramentas e Softwares } \\
\text { de Gestão }\end{array}$ & $\begin{array}{l}\text { Não utilização de softwares complexos; utilização de } \\
\text { ferramentas limitadas. }\end{array}$ \\
\cline { 2 - 3 } & 5. Comunicação & $\begin{array}{l}\text { Comunicação próxima e informal; baixo nível de } \\
\text { formalização; e utilização de celular. }\end{array}$ \\
\hline Encerramento & 6. Avaliação de Resultados & $\begin{array}{l}\text { Alto grau de engajamento do proprietário com os } \\
\text { objetivos e os resultados do projeto; baixo grau de } \\
\text { avaliação quantitativa da performance do projeto; } \\
\text { avaliação do escopo e aprendizagem limitada. }\end{array}$ \\
\hline
\end{tabular}

Figura 2. Modelo pré-existente para gerenciamento de projetos em pequenas empresas Fonte: Adaptada de Kozlowski e Matejun (2016).

Quando há um gerenciamento de projetos adequado, as ações podem ser implementadas de forma flexível e racionalizada, atendendo às necessidades das 
PME, com o desenvolvimento de novos produtos, a proposta de soluções e a busca de vantagem competitiva (Kozlowski \& Matejun, 2016; Turner et al., 2012). O foco, então, está nos resultados, não nos impactos que tal gestão pode ocasionar nos negócios (Pollack \& Adler, 2016).

Embora os estudos sobre o gerenciamento tenham a tendência de se concentrar nos megaprojetos, resultantes de mais investimentos e de maiores resultados (Pollack \& Adler, 2016), não se pode ignorar as vantagens dos projetos das PME, que trazem melhoria na qualidade, maior eficiência, implementação rápida de novas demandas, desenvolvimento de novos produtos, retorno financeiro (Kozlowski \& Matejun, 2016), além do auxílio nos processos de gestão da organização (Martens et al., 2014; Oliveira \& Figueiredo, 2016; Sella \& Grzybovski, 2011; Silva \& Matamoros, 2015).

\section{METODOLOGIA}

Com intenção de caracterizar os conceitos do gerenciamento de projetos nas PME, este estudo apresentou uma abordagem exploratória, com caráter teórico-descritivo, por meio de um estudo bibliométrico de cocitação, associado à análise lexical e à CHD.

A bibliometria permite ao pesquisador consumir uma quantidade elevada de materiais, publicados no campo científico, e, a partir dessas coletas, desenvolver uma visão sistematizada e resumida, que auxilia o entendimento a respeito dos trabalhos que compõem o campo de pesquisa (Quevedo-Silva, Santos, Brandão, \& Vils, 2016).

Já, pela perspectiva de cocitação, é possível identificar autores e trabalhos citados em pares, dentro de um grupo de artigos que compõem o campo de estudos, bem como os interesses e os padrões intelectuais, estabelecidos pelas relações entre eles (Small, 1973), classificando, com isso, aqueles com maior relevância (Zupic \& Čater, 2015). Neste estudo, para o levantamento de dados, o primeiro passo foi usar o filtro de artigos pelo tópico "Project management and small medium business", na Web of Science, base escolhida por sua credibilidade como repositório de artigos, com elevado fator de impacto na comunidade científica (Mugnaini \& Strehl, 2008), e por ela ter sido utilizada em pesquisas anteriores, com a mesma finalidade (Bernardo, Ramos, \& Vils, 2018).

Após a coleta dos primeiros resultados, houve dois refinamentos: pelas áreas "business and management", haja vista o interesse da pesquisa; e somente por artigos. Extraiu-se, então, os artigos com extensão “.txt”, sem formatação, que foram inseridos no software BibExcel, para a continuidade da bibliometria de cocitação. 
No BibExcel, os arquivos ".txt" gerados foram lidos, e se procedeu à aplicação dos passos necessários à sua transformação para os formatos ".doc" e ".cit", que, por sua vez, seguiram para a análise de coocorrência, por meio da seleção, pela frequência de citação >=2, com base na Lei de Lotka, 211 artigos(Figura 3).

Pelos princípios da Lei de Lotka, somente os arquivos com maior frequência de citação, responderão pelo campo de estudos (Quevedo-Silva et al., 2016). Por conta desse novo corte, 70 artigos resultaram da cocitação, os quais seguiram para download, com fins de extração dos resumos, dos resultados e das conclusões. Ressalta-se, contudo, que, destes, 25 foram descartados, devido ao acesso ao texto não ter sido localizado.

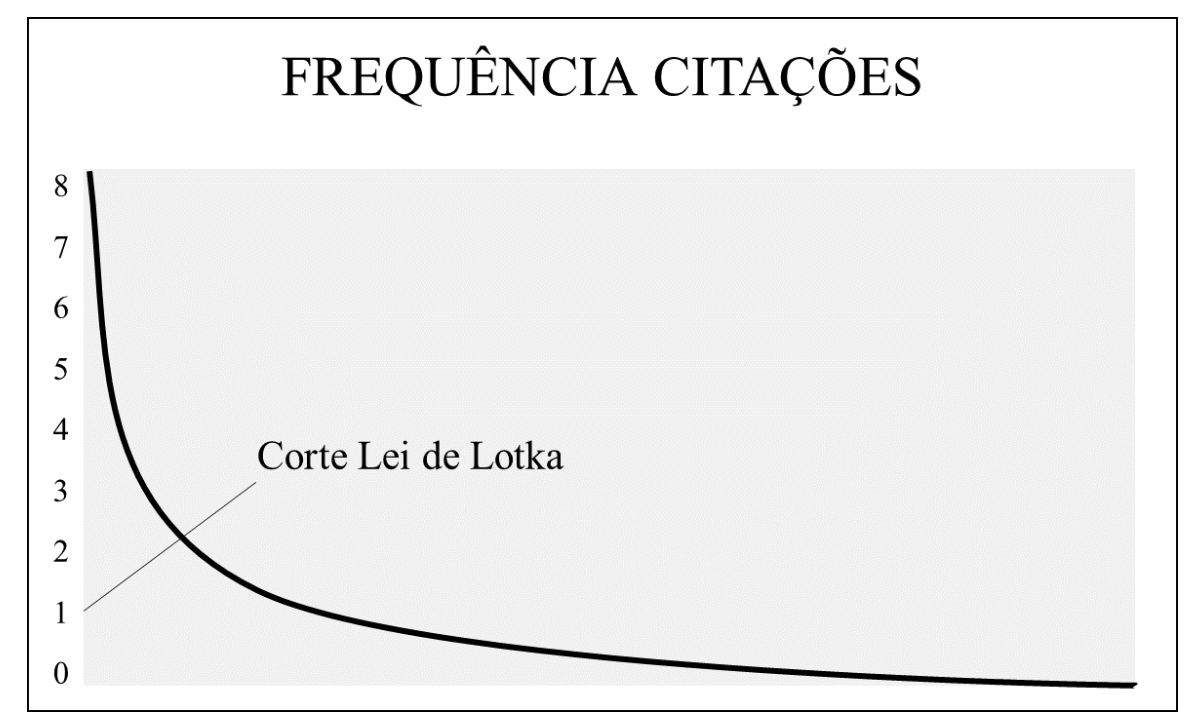

Figura 3. Corte da Lei de Lotka para Gerenciamento de Projetos em PME Fonte: Elaborada pelos autores (2020).

Os resumos, as análises e os resultados dos 45 artigos identificados foram extraídos e submetidos à CHD, no software Alcaceste - Iramuteq (Camargo \& Justo, 2013). Optouse pela utilização desses fragmentos, pois eles representam as partes dos textos em que os autores expressam o seu entendimento do tema, sem, necessariamente, fazer uso de citações.

Essa análise permitiu a compreensão de vocábulos específicos, que se interrelacionaram nas publicações, bem como a estatística do que os autores quiseram expressar em suas pesquisas (Camargo \& Justo, 2013). Dessa forma, classes de segmentos de textos foram geradas e lidas, a fim de encontrar os conceitos necessários à elaboração do modelo objetivado por esta pesquisa. Vale ressaltar que "segmentos de texto" são trechos que auxiliam no entendimento do vocabulário resultante da CHD 
(Camargo \& Justo, 2013); e "classes textuais", agrupamentos de segmentos de texto, feitos por frequência de palavras e por semelhança de vocábulos.

Durante a fase de entendimento dos segmentos de textos, resultantes da CHD, foram identificados e selecionados apenas dois artigos voltados ao gerenciamento de projetos para PME, que permitiram a continuidade da pesquisa, não sendo possível, no entanto, a compreensão e o detalhamento das classes textuais.

Uma nova pesquisa, então, foi realizada, na base de dados Google Scholar (Figura 4), pela utilização do filtro "Gerenciamento de projetos em PME”, e excluindo patentes e citações. Nesse levantamento, optou-se pela expressão em português, visando ao alcance de trabalhos com a especificidade de projetos voltados às PME no contexto brasileiro. Dentre os resultados apresentados, por meio da leitura dos títulos, extraiu-se o resumo, os resultados e as conclusões dos artigos (Figura5)

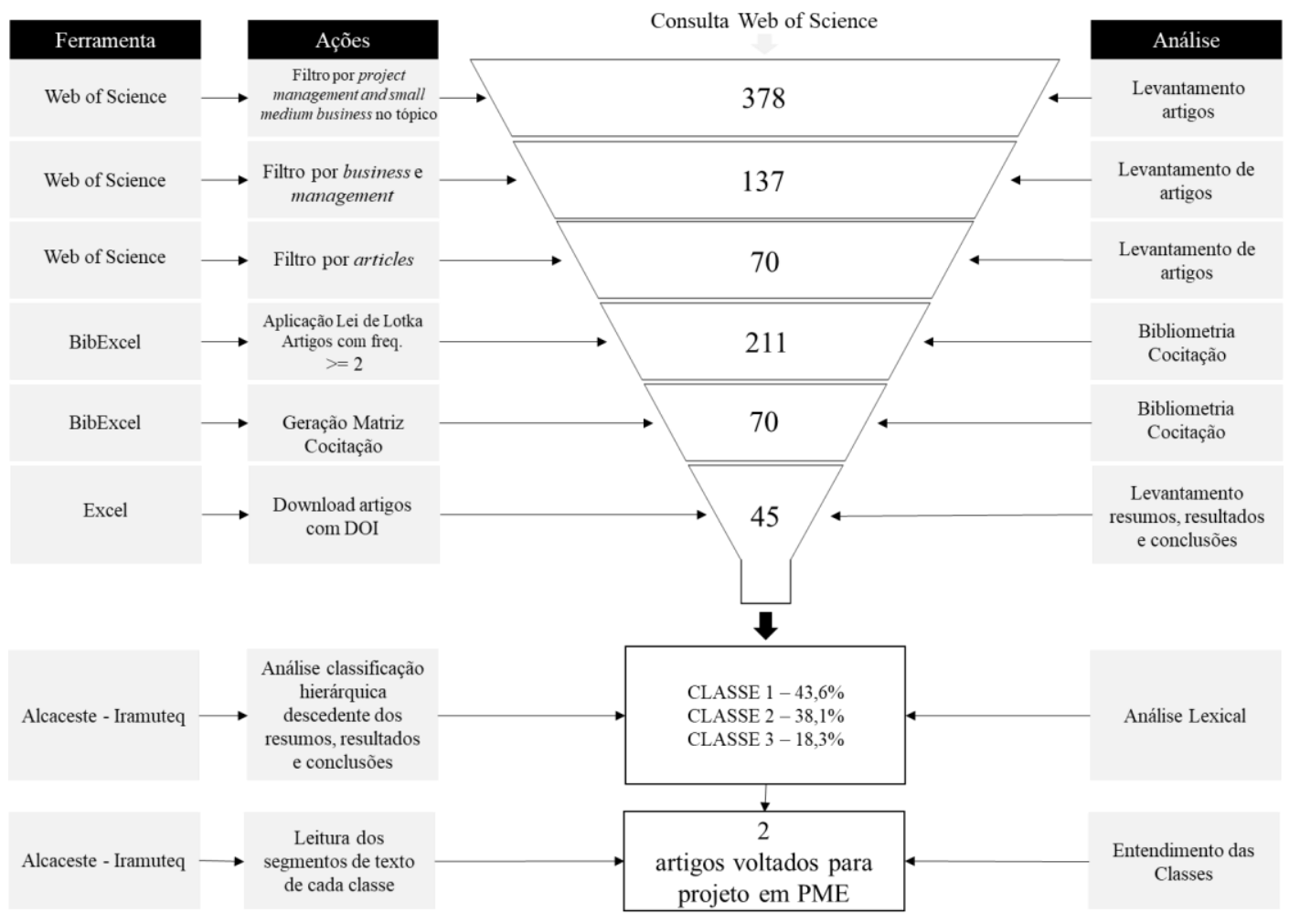

Figura 4. Estudo bibliométrico e lexical para Gerenciamento de Projetos na Web of Science Fonte: Elaborada pelos autores (2020). 
Proposição de um Modelo de Gerenciamento de Projetos para Pequenas e Médias Empresas (PME)

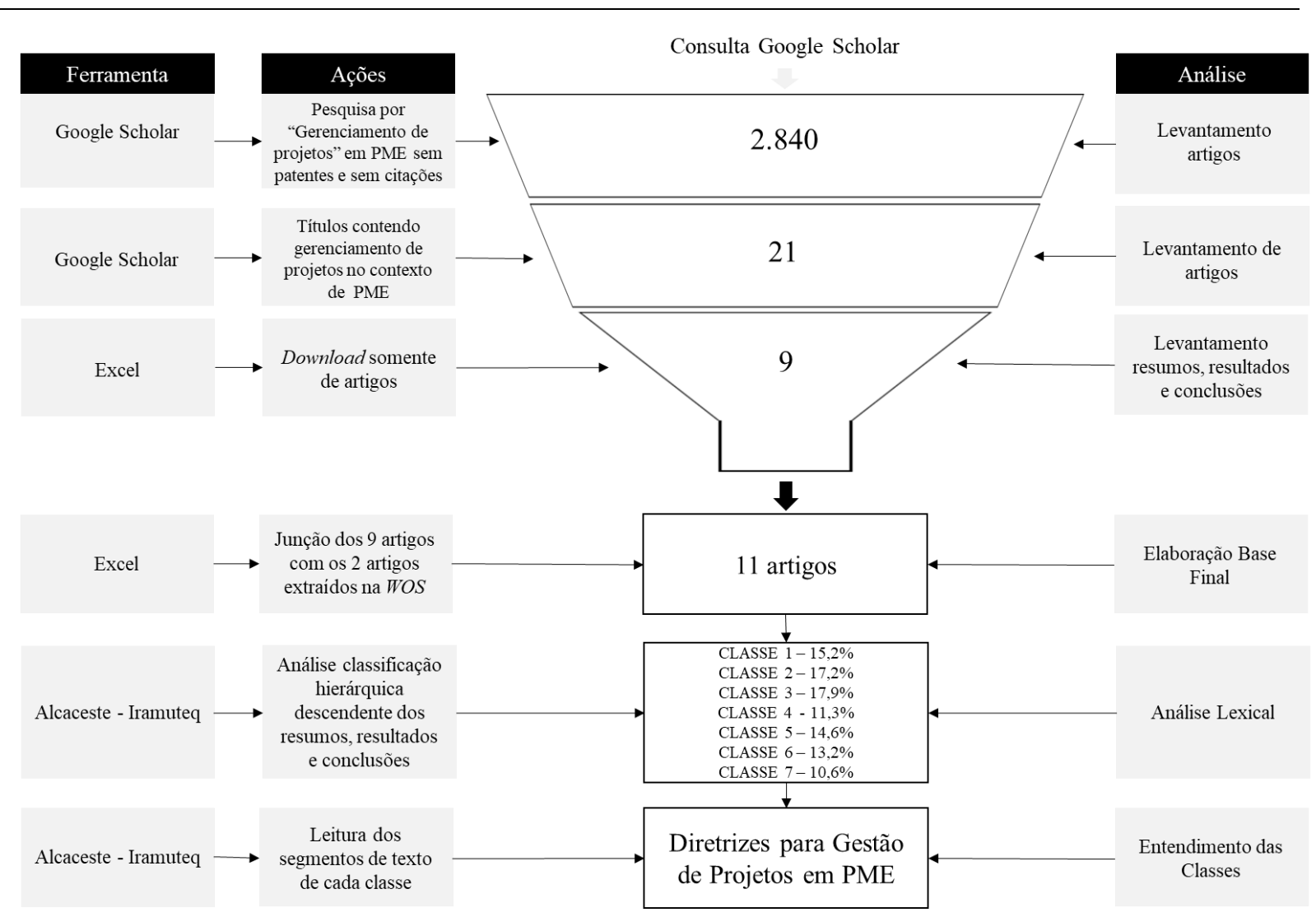

Figura 5. Estudo bibliométrico e lexical para Gerenciamento de Projetos no Google Scholar Fonte: Elaborada pelos autores (2020).

Os textos dos nove artigos extraídos do Google Scholar (Tabela 1) foram submetidos a uma nova análise lexical, em conjunto com os textos dos dois artigos resultantes da pesquisa na Web of Science. Devido à quantidade de artigos localizados nessas bases de dados, houve uma caracterização exploratória, para o entendimento do estado atual do conhecimento sobre o gerenciamento de projetos em PME. Então, a partir da leitura dos segmentos de textos das classes resultantes, foi possível propor um modelo. 
Tabela 1

\section{Artigos resultantes das análises}

\begin{tabular}{|c|c|}
\hline Autor & Artigo (Journal) \\
\hline Turner et al. (2010) & $\begin{array}{l}\text { Project management in small to medium-sized enterprises (International Journal of Project } \\
\text { Management). }\end{array}$ \\
\hline Turner et al. (2012) & $\begin{array}{l}\text { Project management in small to medium-sized enterprises: Tailoring the practices to the size of } \\
\text { company (Management Decision). }\end{array}$ \\
\hline Carvalho e Mello (2012) & $\begin{array}{l}\text { Aplicação do método ágil scrum no desenvolvimento de produtos de software em uma pequena } \\
\text { empresa de base tecnológica (Gestão \& Produção). }\end{array}$ \\
\hline Castro e Moraes (2015) & $\begin{array}{l}\text { Uso de modelos de maturidade em gestão de projetos em uma pequena empresa de TI (Anais do IV } \\
\text { SINGEP). }\end{array}$ \\
\hline Jucá Júnior et al. (2010) & $\begin{array}{l}\text { Maturidade em gestão de projetos em pequenas empresas desenvolvedoras de software do Polo de } \\
\text { Alta Tecnologia de São Carlos (Gestão \& Produção). }\end{array}$ \\
\hline Martens et al. (2014) & $\begin{array}{l}\text { Gerenciamento de projetos em micro e pequenas empresas (Revista Pensamento Contemporâneo } \\
\text { Em Administração). }\end{array}$ \\
\hline Oliveira e Figueiredo (2016) & $\begin{array}{l}\text { Gestão de projetos em pequenas e médias empresas: Pesquisa ação em uma empresa da indústria } \\
\text { da moda. (Workshop de Gestão, Tecnologia Industrial e Modelagem Computacional). }\end{array}$ \\
\hline Sella e Grzybovski (2011) & $\begin{array}{l}\text { Modelo PMBOK/PMI para gestão de projetos nas micro e pequena empresas: um estudo de caso } \\
\text { (Revista Economia \& Gestão). }\end{array}$ \\
\hline Silva e Matamoros (2015) & $\begin{array}{l}\text { Gestão de projetos como ferramenta estratégica para pequenas empresas. (Revista de Ciências } \\
\text { Gerenciais). }\end{array}$ \\
\hline Souza et al. (2014) & Fatores críticos para gerenciar projetos em empresas de pequeno porte (Anais do VI Simprod). \\
\hline $\begin{array}{l}\text { (Artigo sem relevância ao final da análise lexical e } \\
\text { CHD) }\end{array}$ & $\begin{array}{l}\text { A importância da Gestão do Escopo para uma boa gestão do Projeto em Micro e Pequenas } \\
\text { Empresas. }\end{array}$ \\
\hline
\end{tabular}

Fonte: Elaborada pelos autores (2020). 
Proposição de um Modelo de Gerenciamento de Projetos para Pequenas e Médias Empresas (PME)

\section{RESULTADOS E ANÁLISES}

Para a construção desta pesquisa, foram necessárias duas buscas de artigos voltados ao gerenciamento de projetos para PME: a primeira, em inglês, resultando em dois artigos; e a segunda, em português, incorporando mais nove trabalhos, totalizando 11 artigos, que compuseram a base do modelo proposto.

Tal achado suscita o debate se o tema "gerenciamento de projetos", no contexto das PME, tem atraído maior atenção em comunidades científicas de países lusófonos, em especial, o Brasil. Discute-se, também, a partir disso, a possibilidade de países em desenvolvimento apresentarem maior interesse no estudo de pequenas organizações do que países desenvolvidos, os quais já possuem grandes corporações como base de sua economia. Os resultados da análise lexical e da CHD, referentes aos textos extraídos dos 11 artigos selecionados, geraram 5.887 ocorrências e 163 segmentos de textos, categorizadas em sete classes textuais (Figura 6).

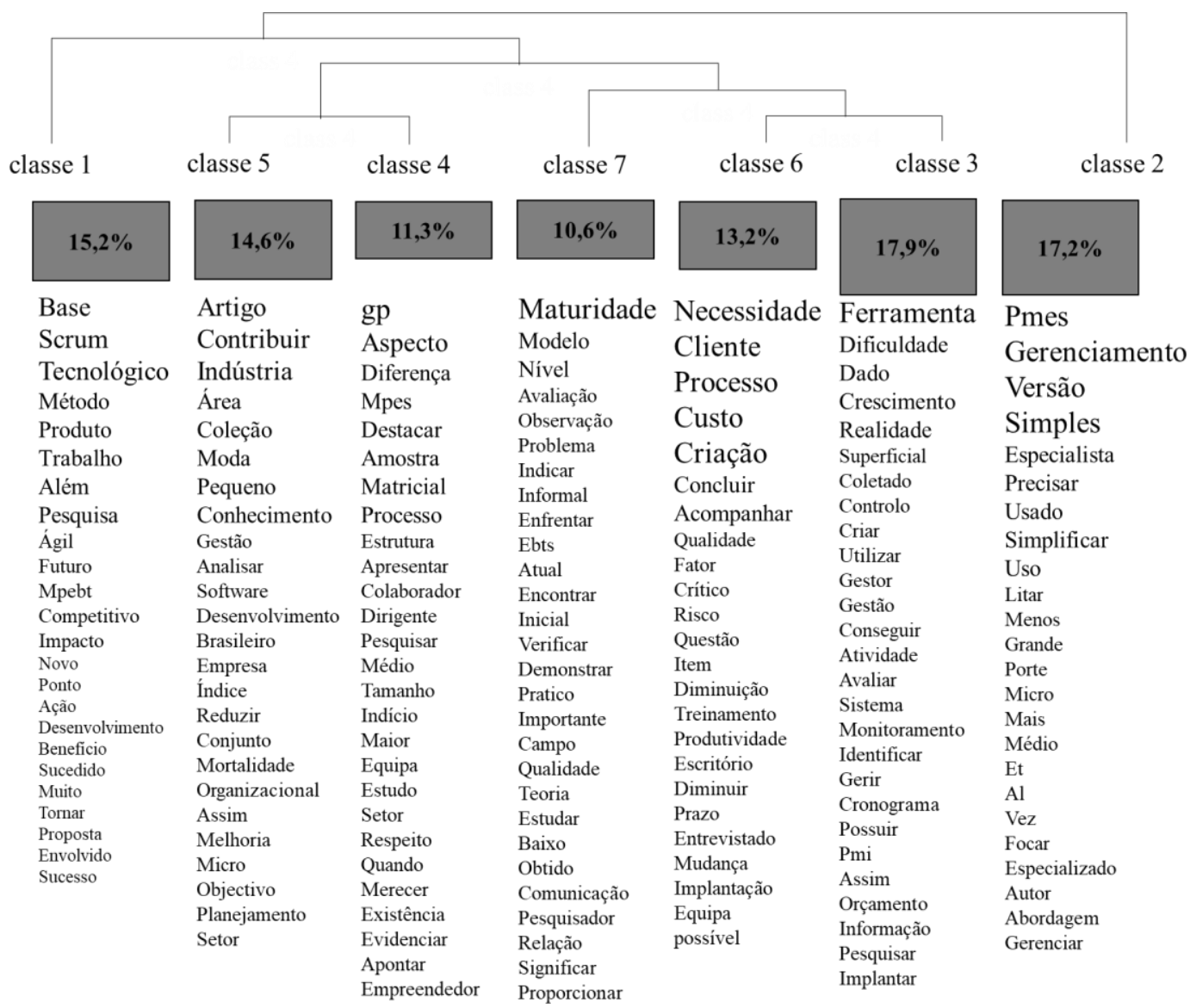

Figura 6. Resultado análise lexical

Fonte: Elaborada pelos autores (2020).

Rev. de Empreendedorismo e Gest. Pequenas Empres. | São Paulo, v.9 | n.3 | p. 384-407 | Maio/Ago. 2020. 
Para a compreensão de cada uma das classes, realizou-se a extração e a subsequente leitura de seus respectivos segmentos de textos, com a pontuação de escore absoluta e número máximo de ST de 50.

A Classe 1, que tratou dos métodos ágeis (mais flexíveis e adaptativos do que os tradicionais) do gerenciamento de projetos e da sua importância para as PME, representou $15,2 \%$ do corpus textual. Dentre os benefícios destacados, estão: a melhoria da comunicação e a colaboração entre os membros da equipe; a diminuição do risco; e o aumento da eficiência do cronograma de execução dos projetos (Carvalho \& Mello, 2012). Nessa classe, foram identificados: a flexibilidade do projeto, em relação ao contexto da empresa; os processos de controle do cronograma, para o devido planejamento do tempo da execução; a gestão da comunicação, entre todos os envolvidos; e a gestão dos riscos, relacionados aos aspectos que podem influenciar os resultados do projeto.

A Classe 2, representativa de $17,2 \%$ do corpus textual, abordou as características de simplificação e de gerenciamento de projetos por pessoas não especialistas, nas PME. Constatou-se a ampla utilização do gerenciamento de projetos nessas organizações, sendo que o empreendedor atua como gerente de projetos, cuidando da implementação das técnicas simples de coleta de dados e das tomadas de decisões (Turner et al., 2010, 2012; Jucá Júnior et al., 2010). Nessa classe, foi apontada a premissa de utilização do método simplificado, com baixo custo de implantação, além de processos desburocratizados, para a execução dos projetos dentro da empresa, resultando em maior facilidade de gerenciamento por pessoas não especialistas. Quanto aos processos, notou-se a necessidade de fazer o controle dos pedidos essenciais do projeto (ou requisitos), alinhado ao orçamento disponível. Como benefício, as pessoas não especialistas desenvolvem as competências necessárias para o gerenciamento dos projetos.

As Classes 3 e 6 se inter-relacionaram, representando, juntas, 31,10\% do corpus textual. Elas destacaram a importância, para as PME, das ferramentas e das técnicas de gerenciamento de projetos, associadas ao gerenciamento de custos, à comunicação e aos orçamentos. As maiores dificuldades observadas, quanto ao gerenciamento de projetos, estão nas ferramentas e técnicas, bem como nas habilidades necessárias do gerente de projetos para a sua condução (Souza et al., 
Proposição de um Modelo de Gerenciamento de Projetos para Pequenas e Médias Empresas (PME) 2014). Ainda assim, percebe-se que a gestão de projetos é feita, e as empresas mantêm-se em crescimento (Silva \& Matamoros, 2015), visto que as ferramentas e técnicas utilizadas permitem a eles se antecipar aos problemas (Souza et al., 2014). Essas classes indicaram que o projeto deve ter mais foco nas pessoas do que nas ferramentas, o que exige um gerenciamento adequado da comunicação entre os envolvidos, e orçamento disponível. A junção entre objetivo e processos desenvolve as competências de gestão de projetos.

Impulsionadas pela competição de preços e pela imposição de qualidade pelos consumidores das PME, grandes mudanças nos processos de gestão ocorreram no século XX (Sella \& Grzybovski, 2011). Por conta disso, quando há um hiato no gerenciamento de projetos de uma PME, é automaticamente criada uma barreira, que Ihe impede a realização e a entrega de projetos mais complexos. Isso limita a sua capacidade (Jucá Júnior et al., 2010), mas mantém essas empresas ativas (Souza et al., 2014).

Entre os fatores de sucesso do gerenciamento de projetos em PME, estão a gestão de custos e orçamentária (Souza et al., 2014), a comunicação e os riscos (Carvalho \& Mello, 2012; Martens et al., 2014), além do treinamento em gestão de projetos para pequenos empresários (Castro \& Moraes, 2015).

As Classes 4 e 5 também se inter-relacionaram, representando 25,9\% do corpus textual. Elas denotam a importância do gerenciamento de projetos para os processos de aprendizagem da gestão do negócio (Martens et al., 2014; Oliveira \& Figueiredo, 2016; Sella \& Grzybovski, 2011; Silva \& Matamoros, 2015). Nesse sentido, constatouse que as PME têm maior alinhamento com processos de gerenciamento de projetos, quando a estrutura é matricial, especialmente em empresas de tecnologia (Martens et al., 2014).

Por fim, a Classe 7 , que representa $10,6 \%$ do corpus textual, indica que os aspectos de maturidade, em gerenciamento de projetos, não parecem compor um fator crítico, tendo em vista as práticas mais informais e flexíveis das PME.

Os resultados de cada classe foram utilizados para a elaboração da Proposta de Gerenciamento de Projetos em PME (Figura 7), o que, por sua vez, indica um avanço em relação ao modelo sugerido por Kozlowski e Matejun (2016). Com relação às características, o modelo destacou como os projetos em PME precisam ser 
desburocratizados e flexíveis, a fim de atender a todas as pessoas envolvidas. Em termos de processos, foi possível descobrir a importância da gestão dos requisitos do projeto, bem como das comunicações e dos riscos a serem tratados ao longo do seu desenvolvimento. Por fim, como benefícios, destacou-se como os proprietários podem, além de alcançar resultados financeiros com os projetos, desenvolver competências de gestão, capazes de os auxiliar em outras áreas de conhecimento do negócio.

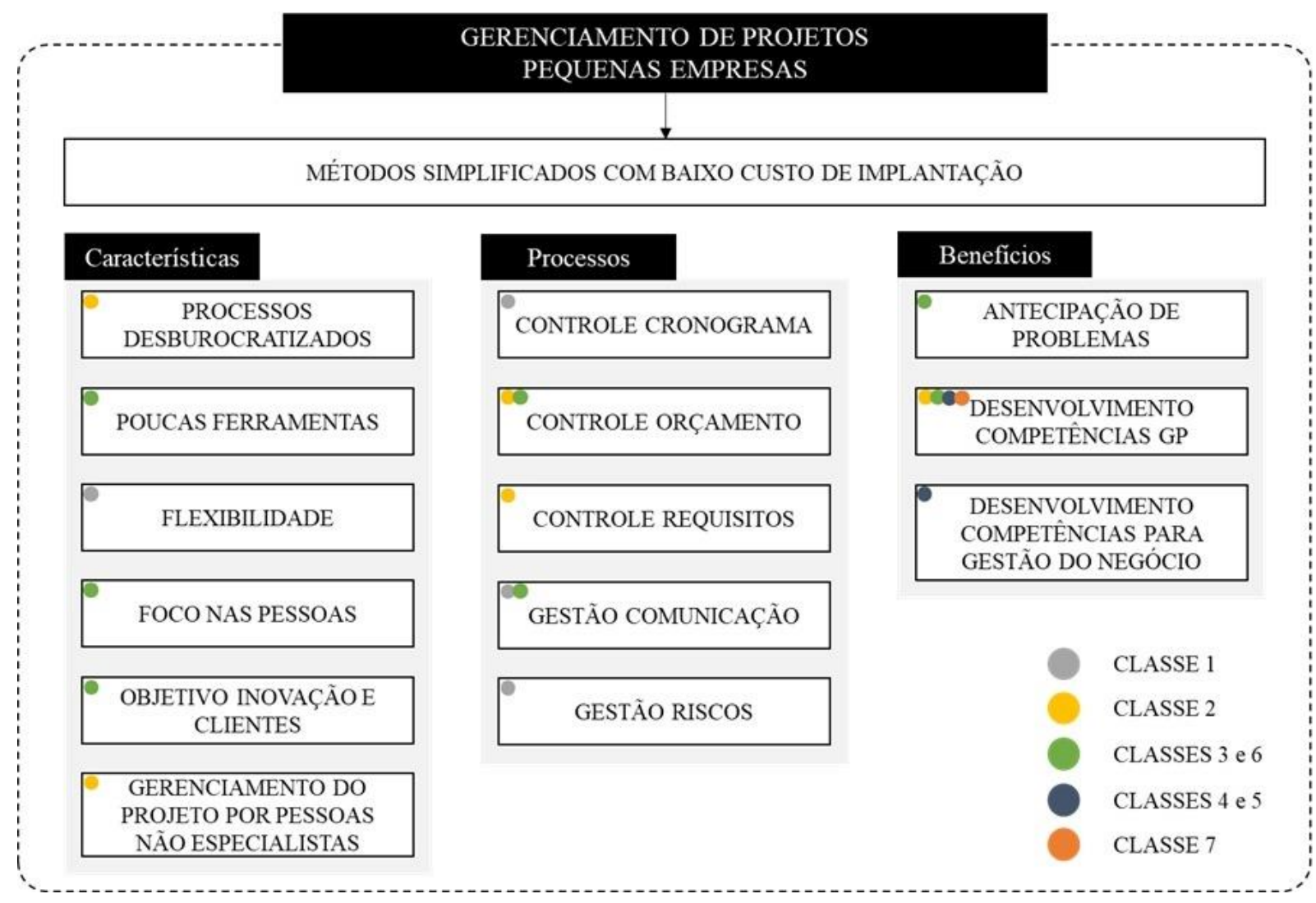

Figura 7. Modelo proposto para Gerenciamento de Projetos em PME, a partir da análise lexical Fonte: Elaborada pelos autores (2020).

\section{CONCLUSÃO}

Para entender o estado atual do conhecimento e nortear uma possível instanciação de métodos de gerenciamento de projetos, adequados às PME, foi proposto um modelo, por este estudo, pautado no levantamento, em bases de dados (Web Science e Google Scholar), de 11 publicações existentes sobre o tema. Os resumos, as análises e os resultados, extraídos desses artigos, foram submetidos à CHD, pelo software Alcaceste - Iramuteq (Camargo \& Justo, 2013), resultando na 
Proposição de um Modelo de Gerenciamento de Projetos para Pequenas e Médias Empresas (PME) identificação, por meio de uma abordagem exploratória e qualitativa, das relações estabelecidas entre os conceitos e os artigos estudados.

Este estudo evidenciou como a bibliometria, atrelada à análise lexical, com CHD, pode ajudar na construção de um modelo conceitual de gerenciamento de projetos. Por essa razão, ele oferece uma contribuição teórica para a literatura sobre o assunto, evoluindo o modelo de Kozlowski e Matejun (2016); e uma contribuição prática, já que esse modelo pode ser contextualizado nas PME, incentivando a implementação de novas iniciativas, de forma padronizada e clara, pelos sócios proprietários.

A partir dos resultados alcançados, foi possível acrescentar ao modelo préexistente de projetos para PME, proposto por Kozlowski e Matejun (2016): (1) as motivações e objetivos do projeto voltados à inovação, com foco no cliente; (2) as características de desburocratização, de flexibilidade, de foco nas pessoas e do gerenciamento por pessoas não especialistas; (3) a premissa de que modelos como esse precisam ser simplificados, com baixo custo de implementação, algo que não tinha sido previsto anteriormente; (4) a gestão do cronograma, de requisitos e dos riscos envolvidos no projeto, nos processos de gerenciamento; e (5) a capacidade de as PME se anteciparem aos problemas, além do desenvolvimento de competências em gerenciamento de projetos e na gestão do negócio.

A limitação da pesquisa se deu pela escolha do levantamento teórico, feito por meio da bibliometria de cocitação, que apresenta como resultados artigos amplamente difundidos no estudo do tema, mas que exclui os mais recentes, por eles ainda não terem recebido um volume considerável de citações.

Como sugestão, novos estudos qualitativos, com empresas que permitam a implementação desse modelo, podem detalhar as características, os processos e os benefícios envolvidos. Além disso, considerando que o modelo proposto partiu da literatura, cabe o desenvolvimento de estudos de natureza empírica, para verificar a viabilidade de sua utilização em campo. Essa pode ser uma forma de defender a coerência do modelo com a realidade das PME, principalmente se comparada a adoção de modelo semelhante, no contexto de empresas de grande porte. Ademais, novas investigações podem ser elaboradas, a fim de agregar maior clareza e relevância à literatura relativa ao gerenciamento de projetos nas $\mathrm{PME}$, em países em desenvolvimento, como o Brasil. 


\section{REFERÊNCIAS}

Adair, P., \& Adaskou, M. (2015). Trade-off theory vs. Pecking order theory and the determinants of corporate leverage: Evidence from a panel data analysis upon french SMEs (2002-2010). Cogent Economics and Finance, 3(1), 1-12.

Almeida, J. M. S. de, Costa, P. R. da, Braga Junior, S. S., \& Porto, G. S. (2018). Capacidade Relacional e Desenvolvimento de Novos Produtos em Pequenas Empresas de Base Tecnológica. Regepe - Revista de Empreendedorismo e Gestão de Pequenas Empresas, 7(3), 141-166.

Bernardo, E. G., Ramos, H. R., \& Vils, L. (2018). Panorama da Produção Científica em Empreendedorismo Rural: Um Estudo Bibliométrico. Regepe - Revista de Empreendedorismo e Gestão de Pequenas Empresas, 8(1), 102-125.

Bos-Brouwers, H. E. J. (2010). Corporate sustainability and innovation in SMEs: Evidence of themes and activities in practice. Business Strategy and the Environment, 19(7), 417435.

Breu, F., Guggenbichler, S., \& Wollmann, J. (2008). Strategic Networks. Vasa, 21(3), 203215.

Camargo, B. V., \& Justo, A. M. (2013). Iramuteq: um software gratuito para análise de dados textuais. Temas em Psicologia, 21(2), 513-518.

Carvalho, B. D., \& Mello, C. H. P. (2012). Aplicação do método ágil scrum no desenvolvimento de produtos de software em uma pequena empresa de base tecnológica. Gestão \& Produção, 19(3), 557-573.

Castro, L. M., \& Moraes, R. D. O. (2015). Uso de modelos de maturidade em gestão de projetos em uma pequena empresa de TI. Anais do Simpósio Internacional de Gestão de Projetos, Inovação e Sustentabilidade - SINGEP, São Paulo, SP, Brasil, 4.

Cohen, W. M., \& Levinthal, D. A. (2009). Absorptive Capacity: A New Perspective on and Innovation Learning. Administrative Science Quarterly 35(1), 128-152. 
Proposição de um Modelo de Gerenciamento de Projetos para Pequenas e Médias Empresas (PME) Dobbs, M., \& Hamilton, R. T. (2007). Small business growth: recent evidence and new directions. International Journal of Entrepreneurial Behavior \& Research, 13(5), 296-322.

Jamali, D., Zanhour, M., \& Keshishian, T. (2009). Peculiar strengths and relational attributes of SMEs in the context of CSR. Journal of Business Ethics, 87(3), 355-377.

Jenkins, H. (2006). Small business champions for corporate social responsibility. Journal of Business Ethics, 67(3), 241-256.

Jenkins, H. (2009). A "business opportunity" model of corporate social responsibility for small - and medium - sized enterprises. Business Ethics-a European Review, 18(1), 21 36.

Jucá Júnior, A. S., Conforto, E. C., \& Amaral, D. C. (2010). Maturidade em gestão de projetos em pequenas empresas desenvolvedoras de software do Polo de Alta Tecnologia de São Carlos. Gestão \& Produção, 17(1), 181-194.

Köksal, B., \& Orman, C. (2014). Determinants of capital structure: evidence from a major developing economy. Small Business Economics, 44(2), 255-282.

Kozlowski, R., \& Matejun, M. (2016). Characteristic features of project management in small and medium-sized enterprises. E+M Ekonomie a Management, 19(1), 33-48.

Kwak, Y. H., \& Anbari, F. T. (2009). Analyzing project management research: Perspectives from top management journals. International Journal of Project Management, 27(5), 435446.

Leone, N. M. D. C. P. (1991). A dimensão física das pequenas e médias empresas (PME'S): À procura de um critério homogeneizador. Revista de Administração de Empresas, 31(2), 53-59.

Lewis, Virginia L., \& Churchill, N. C. (1983). The Five Stages of Small Business Growth. Harvard Business Review, 61(3), 30-50. 
Martens, C. D. P., Belfort, A. C., Carneiro, K. D. A., \& Martens, M. L. (2014). Gerenciamento de projetos em micro e pequenas empresas. Revista Pensamento Contemporâneo em Administração, 8(3), 151-171.

Mugnaini, R., \& Strehl, L. (2008). Recuperação e impacto da produção científica na era Google: uma análise comparativa entre o Google Acadêmico e a Web of Science. Encontros Bibli: Revista Eletrônica de Biblioteconomia e Ciência Da Informação, 13, 92105.

Oliveira, N. M., \& Figueiredo, P. (2016). Gestão de projetos em pequenas e médias empresas: Pesquisa ação em uma empresa da indústria da moda. In Workshop de Gestão, Tecnologia Industrial e Modelagem Computacional, 2, 78-79.

Packendorff, J. (1995). Inquiring into the temporary organization: New directions for project management research. Scandinavian Journal of Management, 11(4), 319-333.

Pollack, J., \& Adler, D. (2016). Skills that improve profitability: The relationship between project management, IT skills, and small to medium enterprise profitability. International Journal of Project Management, 34(5), 831-838.

Quevedo-Silva, F., Santos, E. B., Brandão, M. M., \& Vils, L. (2016). Estudo Bibliométrico: Orientações sobre sua Aplicação. Revista Brasileira de Marketing, 15(2), 246-262.

Schumacher, E. F. (1983). O negócio é ser pequeno: um estudo de economia que leva em conta as pessoas (Vol. 1). Rio de Janeiro: Zahar.

Sebrae. (2014). Participação das Micro e Pequenas Empresas na Economia Brasileira. Brasília: Sebrae.

Sella, V. T., \& Grzybovski, D. (2011). Modelo PMBOK/PMI para gestão de projetos nas micro e pequena empresas: um estudo de caso. Revista Economia \& Gestão, 11(27), 3666. 
Proposição de um Modelo de Gerenciamento de Projetos para Pequenas e Médias Empresas (PME) Serrasqueiro, Z., \& Caetano, A. (2015). Trade-Off Theory versus Pecking Order Theory: capital structure decisions in a peripheral region of Portugal. Journal of Business Economics and Management, 16(2), 445-466.

Silva, S. A. D. A. M., \& Matamoros, E. P. (2015). Gestão de projetos como ferramenta estratégica para pequenas empresas. Revista de Ciências Gerenciais, 14(20), 27-47.

Simpson, M., Taylor, N., \& Barker, K. (2004). Environmental responsibility in SMEs: Does it deliver competitive advantage? Business Strategy and the Environment, 13(3), 156-171.

Small, H. (1973). Co-citations in the scientific literature: a new measure of the relationship beetween two documents. Journal of the American Society for Information Science, 24(4), 265-269.

Souza, A. M. D., Souza, M. D. V., \& Souza Júnior, A. A. D. (2014). Fatores críticos para gerenciar projetos em empresas de pequeno porte. Anais do Simpósio de Engenharia de Produção de Sergipe - Simprod, São Cristóvão, Sergipe, Brasil, 6.

Spence, L. (1999). Does size matter? The state of the art in small business ethics. Business Ethics: A European Review, 8(3), 163-174.

Subrahmanya, M. B. (2004). Small industry and globalisation: Implications, performance and prospects. Economic and Political Weekly, 39(18), 1826-1834.

Svejvig, P., \& Andersen, P. (2015). Rethinking project management: A structured literature review with a critical look at the brave new world. International Journal of Project Management, 33(2), 278-290.

Tehseen, S., Ahmed, F. U., Qureshi, Z. H., Uddin, M. J., \& Ramayah, T. (2019). Entrepreneurial competencies and SMEs' growth: the mediating role of network competence. Asia-Pacific Journal of Business Administration, 11(1), 2-29.

Turner, R., Ledwith, A., \& Kelly, J. (2010). Project management in small to medium-sized enterprises. International Journal of Project Management, 28(8), 744-755. 
Turner, R., Ledwith, A., \& Kelly, J. (2012). Project management in small to medium-sized enterprises: Tailoring the practices to the size of company. Management Decision, 50(5), 942-957.

Vial, G. (2019). Understanding digital transformation: A review and a research agenda. Journal of Strategic Information Systems, 28(2), 118-144.

Zupic, I., \& Čater, T. (2015). Bibliometric Methods in Management and Organization. Organizational Research Methods, 18(3), 429-472. 